\title{
FREQUENCY AND PULSE LENGTH SCALING OF RF BREAKDOWN IN ACCELERATOR STRUCTURES*
}

\author{
P. B. Wilson, SLAC, Stanford, CA 94309
}

\begin{abstract}
The plasma spot model predicts that small areas of plasma on the order of 10 microns in diameter form in regions of intense $\mathrm{rf}$ electric field near the iris tips of accelerator structures. These plasma spots act as a current source, injecting electrons and ions into the rf field. The model suggests that an observable breakdown event will occur when electrons, extracted from the spot and accelerated, absorb energy faster than it can be supplied by the rf source. This field collapse, which can occur during processing, is not an ultimate limit on accelerating gradient. It is conjectured that surface melting, due to electron and ion back-bombardment at the emitting site, leads to a pseudospark-like discharge which limits the gradient and can produce massive surface damage. Using a statistical model, the dependence on frequency and pulse length of the probability of triggering a plasma spot is examined.
\end{abstract}

\section{INTRODUCTION}

In [1] it is proposed that plasma spots, which form on the metallic surfaces of accelerator structures in regions of intense electric fields, can act as multi-ampere current sources for both electrons and copper ions. In an intense dc field these plasma manifestations, known as cathode spots, act as current sources for kiloampere vacuum arcs. Because of the many practical applications of such dc arcs (high voltage switching, plasma deposition, etc.), the properties of such cathode spots have been intensively studied [2]. Very briefly, a cathode spot is a mushroomlike region of plasma having a density on the order of $10^{20}$ electrons per $\mathrm{cm}^{3}$ and a dimension on the order of 10 microns. A plasma sheath forms at the contact area between the plasma and the metallic surface. This sheath has the properties of a space change limited diode, subjecting the metal surface to an ion bombardment current on the order of $10^{8} \mathrm{~A} / \mathrm{cm}^{2}$ and accelerating a roughly equivalent number of electrons into the plasma. A simple calculation will show that the ions, bombarding the surface with an energy on the order of $20 \mathrm{eV}$, will raise the surface temperature to the melting point in a few nanoseconds. After 30-50ns, the lifetime of a single cathode spot, the subsequent melting will have produced a crater with a diameter of 5-20 microns. In the dc case, such a crater is known to be a well-defined "footprint" of a cathode spot. It is the close similarity between the crater footprints formed in both $\mathrm{dc}$ and rf fields that suggests that the process of formation and the subsequent dynamics for both dc cathode spots and rf plasma spots must have a close similarity. Supporting this conclusion is the fact that field emission, which serves as a trigger for the formation of a dc plasma spot, is essentially the same phenomenon in both $\mathrm{dc}$ and $\mathrm{rf}$ fields. Knobloch and Padamsee have, in fact, simulated the formation of a plasma at a site of field emission in an rf field [3].

A single dc cathode spot produces a current on the order of $10-100 \mathrm{~A}$, and an ion current which is about $10 \%$ of this. The current is roughly constant during the lifetime of the spot. After some $50 \mathrm{~ns}$, the rate of crater growth will have decreased substantially and the expanding plasma cloud will have outrun its source of ionizing electron current. At that point the plasma spot will extinguish. However, secondary spots may be triggered near rough areas around the rim of the crater formed by the primary spot. Clusters of such secondary craters surrounding a central crater are often observed on photomicrographs of surfaces that have been subjected to intensive breakdown events. Thus, in principle, several generations of plasma spots could last for more than $100 \mathrm{~ns}$ and generate a current of several hundred Amperes.

Although the formation and dynamics of the plasma spot itself is essentially a dc phenomenon, the behavior of the electrons and ions following injection into an rf field is dramatically different. The trajectories of electrons and ions from a plasma source are currently being simulated for various TW and SW accelerating structures by Dolgashev and Tantawi [4]. The dynamics of the electron motion are quite complex for the case of emission from the iris tip in an accelerator structure, especially when space charge effects and taken into account. However, the particle motion in such structures has some fundamental similarities to particle motion in the simple geometry of a gap between parallel planes. Analytic results for the parallel-plane case can be used, together with appropriate form factors, to obtain approximate results for more complex structures.

\section{PARTICLE MOTION IN A PARALLEL PLANE GAP}

The equations of motion (non-relativistic) for an electron emitted with zero velocity from a plane at $\mathrm{x}=0$ at a phase angle $\theta_{\mathrm{e}}$ with respect to the crest of an rf field with peak value $\mathrm{E}_{0}$ are

$$
\mathrm{x} / \lambda=\left[\mathrm{E}_{0} \lambda /(2 \pi)^{2} \mathrm{~V}_{\mathrm{e}}\right] \mathrm{x}_{\mathrm{n}} ; \quad \mathrm{v} / \mathrm{c}=\left[\mathrm{E}_{0} \lambda /(2 \pi) \mathrm{V}_{\mathrm{e}}\right] \mathrm{v}_{\mathrm{n}}
$$

where $\mathrm{x}_{\mathrm{n}}=\left[\cos \theta_{\mathrm{e}}-\cos \left(\omega \mathrm{t}+\theta_{\mathrm{e}}\right)-\omega \mathrm{t} \sin \theta_{\mathrm{e}}\right], \quad \mathrm{v}_{\mathrm{n}}=$ $\left[\sin \left(\omega \mathrm{t}+\theta_{\mathrm{e}}\right)-\sin \theta_{\mathrm{e}}\right]$ and $\mathrm{V}_{\mathrm{e}}=\mathrm{mc}^{2} / \mathrm{e}=511 \mathrm{kV}$. For $\mathrm{a}$

\footnotetext{
* Work supported by Department of Energy contract DE-AC03-76SF0051.
} 
wide gap, $x_{n}>2$, it is found that electrons emitted in the phase range $-\pi / 2<\theta_{\mathrm{e}}<0$ always cross the gap and impact on the opposing face, while electrons in the phase range $0<\theta_{\mathrm{e}}<\pi / 2$ return to the emitting surface with an average value $\mathrm{v}_{\mathrm{n}}{ }^{2}=0.62$. For a structure operating near the dark current capture threshold $\mathrm{E}_{0} \lambda=\pi \mathrm{V}_{\mathrm{e}}$ (this threshold is $61 / \mathrm{MV} / \mathrm{m}$ at $11.4 \mathrm{GHz}$ ), and having a gap width of $\lambda / 3$, the normalized gap width is $x_{n}=4.1$ and the average value of $\mathrm{v}_{\mathrm{n}}^{2}$ is about 1.7 for electrons crossing the gap.

\section{CONDITION FOR FIELD COLLAPSE}

The differential equation for field (gradient) collapse in a standing wave structure of $\mathrm{N} \pi$-mode cells is, after turn-on of a plasma spot with dc current $I_{s}$ and rf emitted current $I_{e}$ $=\mathrm{I}_{\mathrm{s}} / 2: \mathrm{G}+\mathrm{T}_{\mathrm{F}}(\mathrm{dG} / \mathrm{dt})=\mathrm{G}_{0}-\mathrm{fI}_{\mathrm{e}}(\mathrm{r} / \mathrm{Q}) \mathrm{Q}_{\mathrm{L}} / \mathrm{N}$. Here $\mathrm{G}_{0}$ is the gradient before spot turn-on, $\mathrm{T}_{\mathrm{f}}$ is the filling time, and $\mathrm{f}$ is the impact energy in electron volts integrated over all the cell surfaces including the entrance and exit irises and all emission phase angles, normalized to the maximum axial energy gain $\mathrm{G}_{0} \mathrm{~g}$ where $\mathrm{g}$ is the cell length:

$$
f=\left(\pi G_{0} g\right)^{-1} \int_{-\pi / 2}^{\pi / 2} d \theta_{e} \int_{S} V_{i m p}\left(\theta_{e}\right) d S .
$$

From the differential equation above, the spot current required to collapse the field in time $t_{c}$ is, for $t_{c}$ small compared to the filling time $\mathrm{T}_{\mathrm{F}}$,

$$
\mathrm{I}_{\mathrm{e}}(\mathrm{SW})=\mathrm{G}_{0} \mathrm{~N} / 2 \mathrm{ft}_{\mathrm{c}} \mathrm{k}_{1}
$$

where $\mathrm{k}_{1}$ is the structure loss factor per meter, $\mathrm{k}_{1}=$ $(\omega / 4)(\mathrm{r} / \mathrm{Q}) \sim 2 \times 10^{14} \mathrm{ohms} / \mathrm{m}$-s at $11.4 \mathrm{GHz}$. A simulation is required to compute an exact value for $\mathrm{f}$, but for typical cases it is estimated to be in the range $0.2-0.4$. For a collapse time of $30 \mathrm{~ns}$, with $\mathrm{f} \sim 0.3$ and $\mathrm{N}=15$ at a gradient of $50 \mathrm{MV} / \mathrm{m}$, the collapse current is $\mathrm{I}_{\mathrm{e}}(\mathrm{SW}) \sim 200$ A. This current could in principle be produced by a cluster of a half dozen plasma spots.

To calculate the required collapse current for a TW structure, we assume the simplest case of a breakdown event in a single cell. Consider such a cell to be a low $\mathrm{Q}$ resonant cavity with two coupling apertures and an external $\mathrm{Q}$ of $\mathrm{Q}_{\mathrm{e}}=(\pi / 3) /\left(\mathrm{v}_{\mathrm{g}} / \mathrm{c}\right)$ for each aperture. Define $\mathrm{X}$ to be the ratio of the power dissipated in the cell to the power emitted through each aperture. Then the power transmitted through the cell (normalized to the local power flow) is $\mathrm{P}_{\mathrm{T}}=4 /(\mathrm{X}+2)^{2}$ and the reflected power is $\mathrm{P}_{\mathrm{R}}=$ $\mathrm{X}^{2} /(\mathrm{X}+2)^{2}$. The dissipated (missing) power is $1-\mathrm{P}_{\mathrm{T}}-\mathrm{P}_{\mathrm{R}}$. In terms of $X$, the required spot current is $I_{e}=G_{0} X / 8 \mathrm{fk}_{1} Q_{e}$, or

$$
\mathrm{I}_{\mathrm{e}}(\mathrm{TW})=3 \mathrm{XG}_{0} \mathrm{v}_{\mathrm{g}} / 4 \mathrm{f} \lambda \mathrm{k}_{1}
$$

To reduce the transmitted power to $10 \%$ requires $\mathrm{R}=4.3$ (the reflected power is $47 \%$ and the missing power is $43 \%$ ). Putting in numbers for $\mathrm{G}_{0}=50 \mathrm{MV} / \mathrm{m}$ and $\mathrm{v}_{\mathrm{g}} / \mathrm{c}=$
0.05 , using $\mathrm{f}=0.3$ and $\mathrm{k}_{1}=2 \times 10^{14} \mathrm{~V} / \mathrm{C}-\mathrm{m}$ as before, we find the required emitted current to be $1.5 \mathrm{kA}$.

If the same current loading is split equally between two adjacent cells, the transmitted power will be reduced to a few percent and the missing power will be greater than $50 \%$ (for current loading in a single cell, the maximum missing power is $50 \%$ ).

\section{CONDITION FOR SURFACE MELTING}

In an rf field, the metal surface surrounding a plasma spot can be raised to the melting point by backbombardment of the electrons emitted in the phase range 0 $<\theta_{\mathrm{e}}<\pi / 2$. From Eq. (1), the average impact energy (eV) over this range is $\left(1 / 8 \pi^{2}\right)\left(\mathrm{E}_{0} \lambda\right)^{2} \mathrm{v}_{\mathrm{n}}{ }^{2}$ (ave)/ $/ \mathrm{V}_{\mathrm{e}}$. For $\mathrm{v}_{\mathrm{n}}^{2}($ ave $)=$ 0.6 , this gives $\mathrm{V}_{\text {imp }}($ ave $)=1.5 \times 10^{-8}\left(\mathrm{E}_{0} \lambda\right)^{2}$. Electron emission from a plasma spot can also produce heating on a opposing surface, such as a neighboring iris tip in an accelerating structure. Although the average impact electron energy will be higher, the impact area will be considerably larger (this is verified by simulations [5]).

The consequences of surface melting in the neighborhood of an existing plasma spot are severe. It seems almost certain that the plasma, initially confined to a small region over a melting crater about 10 microns in diameter, will immediately spread to cover a large fraction of the surrounding liquid layer. We suggest this is the condition for the initiation of a pseudospark discharge [6] capable of producing very high electron and ion currents. Depending on the structure parameters, the electron current may be sufficient to produce immediate field collapse and to quench the discharge. In a worse scenario, the rf source may continue to pump energy into the discharge over a longer time, producing massive surface damage.

The surface temperature rise is readily calculated in two limits, which depend on the relative values of the heat diffusion depth, given by $\mathrm{x}_{\mathrm{D}} \approx 2(\mathrm{Dt})^{1 / 2}$, where $\mathrm{D}=1.1 \mathrm{x}$ $10^{8}$ (microns $)^{2} / \mathrm{s}$ is the diffusivity for copper, and the penetration depth for electrons with impact energy $\mathrm{eV}$, given by $\mathrm{x}_{\mathrm{P}}$ (microns) $\approx 3.4 \times 10^{-8} \mathrm{~V}^{5 / 3}$ [7]. For $\mathrm{x}_{\mathrm{P}}<<\mathrm{x}_{\mathrm{D}}$, the temperature rise is given by $(\Delta \mathrm{T})_{\mathrm{D}}=\left(2 \mathrm{P}_{\mathrm{A}} / \kappa\right)(\mathrm{Dt} / \pi)^{1 / 2}$ $=0.3 \mathrm{P}_{\mathrm{a}} \mathrm{t}^{1 / 2}$, where $\mathrm{P}_{\mathrm{A}}$ is the impact power per unit area and $\kappa=3.8 \mathrm{~W} / \mathrm{cm}-\mathrm{K}$ for copper. For $\mathrm{x}_{\mathrm{P}}$ large compare to $\mathrm{x}_{\mathrm{D}}$, the temperature rise is given by $(\Delta T)_{P}=P_{A} t / C_{V} x_{P}$, where $\mathrm{C}_{\mathrm{V}}=3.4 \mathrm{~J} / \mathrm{cm}^{3}-\mathrm{K}$ for copper.

The impact power is given by $\mathrm{P}=\left(\mathrm{I}_{\mathrm{e}} / 2\right) \mathrm{V}_{\mathrm{imp}}($ ave $)=8 \mathrm{x}$ $10^{-9} \mathrm{I}_{\mathrm{e}}\left(\mathrm{E}_{0} \lambda\right)^{2}$. Since the distance an electron travels per $\mathrm{rf}$ cycle scales (Eq. 1) as $\mathrm{x} \sim \mathrm{E}_{0} \lambda^{2}$, the back-bombardment area will scale as $\mathrm{E}_{0}{ }^{2} \lambda^{4}$. From simulations on a plane surface [8], the rms radius is several hundred microns at 11.4 GHz for $\mathrm{E}_{0}=70 \mathrm{MV} / \mathrm{m}$, giving $\mathrm{A} \sim 1 \times 10^{-16}\left(\mathrm{E}_{0}^{2} \lambda^{4}\right)$. At an iris tip the area might be somewhat larger. Thus the power per unit area scales as $\mathrm{P}_{\mathrm{A}} \sim 1 \mathrm{x} 10^{8}\left(\mathrm{I}_{\mathrm{e}} / \lambda^{2}\right)$ per $\mathrm{m}^{2}$ (independent of $\mathrm{E}_{0}$ !). Putting in some numbers at 11.4 $\mathrm{GHz}$ at $\mathrm{E}_{0}=\mathrm{E}($ surface $)=100 \mathrm{MV} / \mathrm{m}$ and $\mathrm{I}_{\mathrm{e}}=10 \mathrm{~A}$, (a 20 A spot) we calculate $\mathrm{V}_{\text {imp }}($ ave $) \approx 100 \mathrm{kV}$ and $\mathrm{P}_{\mathrm{A}} \approx 1.5 \mathrm{x}$ 
$10^{8} \mathrm{~W} / \mathrm{cm}^{2}$. At $\mathrm{t}=30 \mathrm{~ns}, \mathrm{x}_{\mathrm{D}} \approx 4$ microns and $\mathrm{x}_{\mathrm{P}} \approx 8$ microns. Thus the temperature rise is calculated using $X_{p}$ and is about 1600 degrees. Taking heat diffusion into account will lower the temperature rise.

The preceding expressions can be put together to give the scaling for the melting time as $t_{m} \sim E_{0}{ }^{10 / 3} \lambda^{16 / 3}$. An ultimate gradient limitation is obtained if surface melting occurs in a filling time $t_{m} \sim \lambda^{3 / 2}$. The gradient then scales as $\mathrm{E}_{0} \sim \lambda^{1.15}$. If heat diffusion is taken into account, the exponent will be smaller.

\section{SCALING OF BREAKDOWN RATE}

Using the fact that the formation of a plasma spot is triggered by field emission, we assume that the probability of triggering a plasma spot per unit time is proportional to the field emission current, $\mathrm{p} \sim \mathrm{I}_{\mathrm{FE}} \sim \exp (-1 / \mathrm{BG})$, where the surface field is proportional to the gradient $G$ and $B$ is in some sense an effective beta enhancement factor for all the emitters on the surface. We assume further that the surface has been processed to a level such that the breakdown rate for a very long pulse is essentially zero at a gradient $\mathrm{G}=\mathrm{G}_{0}$. The breakdown probability per unit time can then be written as $\mathrm{p}=\mathrm{A}\left\{\left[\operatorname{exp~B} \mathrm{B}^{-1}\left(1 / \mathrm{G}_{0}-1 / \mathrm{G}\right)\right]-1\right\}$, where $\mathrm{A}$ is in some sense proportional to the effective emitting area. The probability of breakdown in a pulse of length $T_{P}$ is $\mathrm{pT}_{\mathrm{P}}$ (assumed to be small), and the breakdown rate per hour is proportional to this,

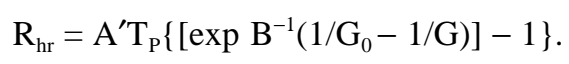

This expression provides a very good fit to measured data on breakdown rates per hour as a function of gradient. Measurements [9] on three different structures gave values for $\mathrm{A}=\mathrm{A}^{\prime} \mathrm{T}_{\mathrm{p}}$ of 2.0, 0.15, 0.30; values for $\mathrm{B}^{-1}$ of 830 , 850,675 ; and values for $\mathrm{G}_{0}$ of $50,56.5,52.5 \mathrm{MV} / \mathrm{m}$.

The above expression predicts a scaling for the breakdown rate as a function of pulse length for a structure at the same state of processing (same $\mathrm{B}$ and $\mathrm{G}_{0}$ ). If $\mathrm{y} \equiv \mathrm{B}^{-}$ ${ }^{1}\left(1 / G_{0}-1 / G\right)$ and $x \equiv A^{\prime} T_{p} / R_{h r}$, then $y=\ln (1+1 / x)$. The dependence of breakdown rate on pulse length is not, therefore, a simple function of pulse length, such as $\mathrm{T}_{\mathrm{P}}^{-1 / 3}$, etc. It depends on the parameters that characterize the surface- A, B and $\mathrm{G}_{0}$ - which in turn also depend on the state of processing.

Expression (4) can also be used to predict the variation of the breakdown rate with frequency. Assume that a structure with identical surface characteristics is scaled with wavelength, $\lambda_{2}=r \lambda_{1}$. Then $A_{2}=r^{2} A_{1}$. The relative gradients for the same pulse length and breakdown rate are then obtained from $\mathrm{y}_{2} / \mathrm{y}_{1}=\left[\ln \left(1+1 / \mathrm{r}^{2} \mathrm{x}_{1}\right)\right] /\left[\ln \left(1+1 / \mathrm{x}_{1}\right)\right]$. It is seen, again, that this is not a "clean" scaling with frequency.

\section{CONCLUSIONS}

Plasma spots form near field emission sites in accelerator structures. The properties of these spots (formation, electron and ion current emission) are similar to those for dc cathode spots.

The electron current from a spot or cluster of spots is accelerated and extracts energy from the rf field.

Complete field collapse requires several hundred amperes in a typical SW structure and several kiloamperes in a typical TW structure.

Electron and ion back-bombardment in the neighborhood of a plasma spot can produce surface melting, resulting in a multi-kiloampere pseudospark-like discharge, which can provide sufficient current to completely cut off power transmission in a TW structure and produce heavy surface damage. In the region of the initial emitting site there should be evidence of surface melting; on an opposing iris tip there should be evidence of surface erosion.

There is no simple scaling $\left(\sim \omega^{\mathrm{n}}\right.$ or $\left.\sim \mathrm{T}_{\mathrm{P}}{ }^{-\mathrm{m}}\right)$ for breakdown gradient as a function of frequency or pulse length.

\section{REFERENCES}

[1] P.B.Wilson, "A Plasma Model for RF Breakdown in Accelerator Structures", LINAC 2000, Monterey, CA, August, 2000.

[2] R. L. Boxman et al. eds., Handbook of Vacuum Arc Science and Technology, (Noyes Publications, Park Ridge, NJ, 1995).

[3] J. Knobloch and H.Padamsee, Particle Accelerators 61, 169-204 (1998).

[4]V. Dolgashev and S. Tantawi, "Simulations of Currents in X-Band Accelerator Structures Using 2D and 3D Particle-in-Cell Codes", this conference.

[5] V. Dolgashev, private communication.

[6] See, for example, Klaus Frank et al.,"'Scientific and Technological Progress of Pseudospark Devices",IEEE Transactions on Plasma Science 27,1008 (1999).

[7] See, for example, R. D. Evans, The Atomic Nucleus, (McGraw-Hill, New York, 1955); Ch. 21.

[8] S. Tantawi, private communication.

[9] C. Adolphsen, private communication. 